\title{
Erratum to: Biosensors Based on Sandwich Assays
}

Fan Xia, Xiaojin Zhang, Xiaoding Lou and Quan Yuan

\section{Erratum to: \\ F. Xia et al. (eds.), Biosensors Based on Sandwich Assays, https://doi.org/10.1007/978-981-10-7835-4}

In the original version of the book, Foreword has been newly included in Frontmatter and authors' affiliations have been updated throughout. The erratum book has been updated with the changes.

The updated online version of this book can be found at https://doi.org/10.1007/978-981-10-7835-4_1 https://doi.org/10.1007/978-981-10-7835-4_2 https://doi.org/10.1007/978-981-10-7835-4_3 https://doi.org/10.1007/978-981-10-7835-4_4 https://doi.org/10.1007/978-981-10-7835-4 5 https://doi.org/10.1007/978-981-10-7835-4_7 https://doi.org/10.1007/978-981-10-7835-4_8 https://doi.org/10.1007/978-981-10-7835-4_10 https://doi.org/10.1007/978-981-10-7835-4_11 https://doi.org/10.1007/978-981-10-7835-4_12 https://doi.org/10.1007/978-981-10-7835-4 